Revue d'histoire de l'Amérique française

Q6 REVUE D.HISTOIRE DE L'AMÉRIQUE FRANÇAISE

\title{
Le poste de La Pointe sur l'île Madeline, tremplin vers le monde franco-anichinabé de la traite des fourrures
}

\section{Nicole St-Onge}

Volume 73, numéro 1-2, été-automne 2019

L’Ouest franco-américain

URI : https://id.erudit.org/iderudit/1068789ar

DOI : https://doi.org/10.7202/1068789ar

Aller au sommaire du numéro

\section{Éditeur(s)}

Institut d'histoire de l'Amérique française

\section{ISSN}

0035-2357 (imprimé)

1492-1383 (numérique)

Découvrir la revue

\section{Citer cet article}

St-Onge, N. (2019). Le poste de La Pointe sur l'île Madeline, tremplin vers le monde franco-anichinabé de la traite des fourrures. Revue d'histoire de l'Amérique française, 73(1-2), 13-43. https://doi.org/10.7202/1068789ar

\section{Résumé de l'article}

Le monde de la traite des fourrures a vu le jour à partir des années 1650 dans le bassin hydrographique des Grands Lacs et du fleuve Saint-Laurent, avec Montréal comme point de jonction entre les Pays d'en Haut et diverses paroisses canadiennes-françaises de la vallée du Saint-Laurent. Les marchandises, les peaux, les populations et les moeurs sociales circulaient dans ce bassin hydrographique ; cette circulation témoignait de l'intense mobilité qui définissait la société vivant de la traite des fourrures. Ce monde a perduré jusqu'au XIX ${ }^{\mathrm{e}}$ siècle, en dépit de profonds changements sociétaux. S'appuyant sur le poste de traite des fourrures de La Pointe comme centre d'observation, l'étude de ces " paysages familiaux » lève le voile sur un monde où les concepts d'identité nationale (ou coloniale) et de frontières étatiques étaient au fond dénués de sens jusqu'au milieu du XIX ${ }^{\mathrm{e}}$ siècle. Les notions autochtones de parenté, liées aux notions franco-catholiques de parenté symbolique (et spirituelle), ont créé toute une série de communautés interreliées, vouées à la traite des fourrures. Celles-ci partageaient des valeurs fondamentales clés et ont permis à une économie et à une société axées sur la traite des fourrures de perdurer et de prospérer pendant plus de deux cents ans.
Tous droits réservés (C Institut d’histoire de l'Amérique française, 2020

Ce document est protégé par la loi sur le droit d'auteur. L’utilisation des services d'Érudit (y compris la reproduction) est assujettie à sa politique d'utilisation que vous pouvez consulter en ligne.

https://apropos.erudit.org/fr/usagers/politique-dutilisation/ 


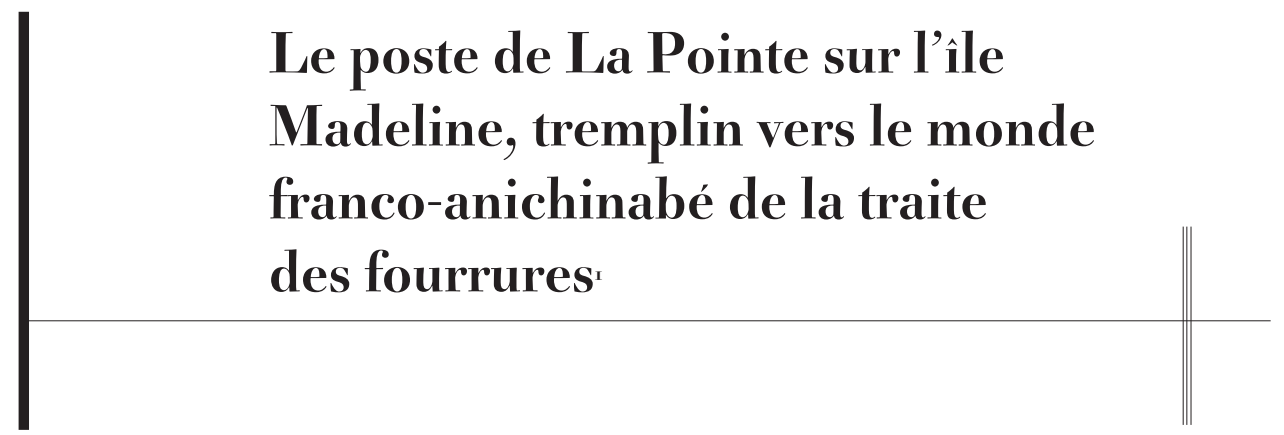

\author{
NiCOLE ST-ONGE \\ Université d'Ottawa
}

RÉsumé • Le monde de la traite des fourrures a vu le jour à partir des années 1650 dans le bassin hydrographique des Grands Lacs et du fleuve Saint-Laurent, avec Montréal comme point de jonction entre les Pays d'en Haut et diverses paroisses canadiennes-françaises de la vallée du Saint-Laurent. Les marchandises, les peaux, les populations et les mœurs sociales circulaient dans ce bassin hydrographique; cette circulation témoignait de l'intense mobilité qui définissait la société vivant de la traite des fourrures. Ce monde a perduré jusqu'au XIXe siècle, en dépit de profonds changements sociétaux. S'appuyant sur le poste de traite des fourrures de La Pointe comme centre d'observation, l'étude de ces «paysages familiaux» lève le voile sur un monde où les concepts d'identité nationale (ou coloniale) et de frontières étatiques étaient au fond dénués de sens jusqu'au milieu du XIX ${ }^{\circledR}$ siècle. Les notions autochtones de parenté, liées aux notions franco-catholiques de parenté symbolique (et spirituelle), ont créé toute une série de communautés interreliées, vouées à la traite des fourrures. Celles-ci partageaient des valeurs fondamentales clés et ont permis à une économie et à une société axées sur la traite des fourrures de perdurer et de prospérer pendant plus de deux cents ans.

ABSTRACT - Beginning in the 1650s, a fur trade world emerged in the Great Lakes-St. Lawrence River watershed, with Montreal as the nexus point linking the Pays d'en Haut to

1. La recherche pour cet article a bénéficié d'une subvention du Conseil de recherches en sciences humaines du Canada (2019-2023) intitulée Fur Trade Kinscapes : La Pointe Wisconsin and the Rise of a Great Lakes-St. Lawrence Watershed, 1650-1850. Celle-ci est détenue conjointement par Robert Englebert et l'auteure. L'auteure tient à remercier Robert Englebert et Galen Perras de leurs commentaires et suggestions sur les versions antérieures du présent article, ainsi que Thierry Simonet, étudiant diplômé, pour avoir dessiné les cartes et diagrammes qui l'illustrent. 
various St. Lawrence Valley French-Canadian parishes. Goods, pelts, peoples and social mores circulated throughout this watershed as manifestations of the intense mobility that defined fur trade society. This world endured into the nineteenth century despite a series of sweeping societal changes. A study of these 'kinscapes', using the fur trade post of La Pointe as locus, reveals a world where until the mid-nineteenth century concepts of national (or colonial) identities and state borders were essentially meaningless. Indigenous notions of kin, linked to French Catholic notions of symbolic kinship (godparents), created a whole series of interlinked fur trade communities that shared sets of key core values and allowed a fur trade economy and society to endure and prosper for over 200 years.

L e présent article concerne le contexte de la traite des fourrures dans la région des Grands Lacs après 1760 . Il porte principalement sur les hommes catholiques, canadiens-français et, par la suite, franco-anichinabés qui, pendant des générations, ont travaillé comme employés salariés d'un commerce des fourrures centré sur Montréal. La vie de ces hommes, dont bon nombre ne sont jamais retournés de façon permanente dans la vallée du Saint-Laurent, sert de point d'entrée dans ce que les historiens ont appelé the French river world - le monde canoté par les Français - la société tournée vers la traite des fourrures qui a vu le jour à Montréal à l'époque de la Nouvelle-France et qui a perduré jusqu'au milieu du XIX siècle ${ }^{2}$.

Même après que de vastes étendues du sud des Grands Lacs furent tombées sous l'égide de l'American Fur Company (AFC) de New York au début du XIX ${ }^{\mathrm{e}}$ siècle, le recrutement d'employés provenant des anciennes paroisses pourvoyeuses de voyageurs de la vallée du Saint-Laurent s'est poursuivi. Entre 1817 et 1840, l'AFC embauche plus de 1100 hommes de la région de Montréal pour travailler dans ses postes de traite des Grands Lacs, du Mississippi supérieur et du Missouri supérieur. Ces hommes de l'AFC ne sont qu'une des dernières manifestations de ce monde créé par une traite des fourrures axée sur Montréal et ancrée dans le bassin versant des Grands Lacs. Ce monde, où le français servait de lingua franca, utilisait à la fois les rites et les rituels catholiques ainsi que les notions françaises et anichinabées de parenté pour parvenir à souder des individus et des familles, malgré une mobilité constante et de grandes distances.

2. Robert Englebert a inventé ce terme dans «Beyond Borders: Mental Mapping and the French River World in North America, 1763-1805 ", thèse de doctorat (histoire), Université d'Ottawa, 2010. 


\section{JUSTIFICATION}

Nombre d'études traitent de certains aspects de la circulation pelletière, une activité de longue durée dans la région des Grands Lacs. Pourtant, peu de chercheurs ont étudié de façon critique la société qui a émergé de cette économie ${ }^{3}$. Des querelles et des débats improductifs centrés sur la question de savoir s'il y a lieu de considérer la population s'adonnant à la traite des fourrures dans la région des Grands Lacs comme "métisse» orientent les débats, universitaires ou publics, en particulier dans le contexte canadien ${ }^{4}$. Le présent article constitue une première incursion dans l'examen des collectivités du passé établies à l'est de la ligne laurentienne de partage des eaux, c'est-à-dire dans le bassin des Grands Lacs et du fleuve Saint-Laurent, où elles se livraient à la traite des fourrures, afin de tracer les contours de ce monde lacustre persistant, fondé sur les pelleteries 5 .

Cet article, comme le vaste projet de recherche qui le sous-tend, se distingue de trois façons des histoires existantes de la région des Grands Lacs. Tout d'abord, il fait fi du découpage chronologique que l'on trouve dans les travaux classiques, puisqu'il tient compte des continuités entre l'époque coloniale française, l'Amérique du Nord britannique et l'après-Révolution américaine. Deuxièmement, en privilégiant l'optique d'un bassin versant des Grands Lacs et du Saint-Laurent élargi, il répond aux récents appels à la création de liens entre les différentes régions du commerce des fourrures des Grands Lacs - États américains des Grands Lacs, Ontario et Québec -

3. Gilles Havard, Histoire des coureurs de bois: Amérique du Nord, 1600-1840 (Paris, Les Indes Savantes, 2016), p. 717-750.

4. La définition généralement acceptée de Métis dans le contexte canadien est qu'ils forment une nouvelle nation distincte, avec une identité autochtone collective fondée sur une origine commune dans l'économie basée sur la traite des fourrures qui a pris naissance à la fin du XVIII ${ }^{\mathrm{e}}$ et au début du XIX siècle dans les grandes plaines du nord des États-Unis et du Canada. Les Métis ne sont pas définis par le double héritage de leurs ancêtres maternels ou paternels, mais par leur identité nationale, leur histoire, leur culture, leur langue, leurs traditions politiques et économiques communes. Les débats actuels portent sur la question de savoir si les populations issues de la traite des fourrures avaient un sentiment commun et distinct d'identité autochtone collective à l'est de la région des Plaines du Nord, et si ces populations devraient également être qualifiées de métisses. Voir Adam Gaudry et Darryl Leroux, «White Settler Revisionism and Making Metis Everywhere: The Evocation of Métissage in Quebec and Nova Scotia ", Critical Ethnic Studies, 3, 1 (printemps 2017), p. 116-142.

5. La ligne laurentienne de partage des eaux sépare l'orientation de l'écoulement des eaux dans l'est et le sud du Canada vers le golfe du Saint-Laurent, d'une part, et, dans le nord du Midwest des États-Unis et du Canada vers la baie d'Hudson, d'autre part. Dans le sud de la région du lac Supérieur, Grand Portage chevauche la hauteur des terres - le partage des eaux -, ce qui offre ainsi un passage entre ces deux bassins hydrographiques essentiels à la traite des fourrures. Carolyn Gilman, The Grand Portage Story (St. Paul, Minnesota Historical Society, 1991), p. 1-23. 
qui sont encore souvent étudiées séparément ${ }^{6}$. Ce n'est qu’une fois cette macroanalyse terminée que les chercheurs seront en mesure de poursuivre une discussion éclairée sur les convergences et les divergences entre les caractéristiques des collectivités vivant de la traite des fourrures autour des Grands Lacs et celles de leurs homologues du bassin versant de la baie d'Hudson, les Métis des Plaines. Troisièmement, en abordant l'histoire de ces collectivités et de ces familles de commerçants de fourrures sous un angle élargi, axé sur les Pays d'en Haut, nos travaux s'alignent sur l'historiographie récente qui tente d'étudier l'histoire des Grands Lacs dans la perspective spatiotemporelle des Anichinabés, peuple dont les influences démographiques et hégémoniques se font sentir dans cette région jusque tard dans le XIX ${ }^{\mathrm{e}}$ siècle?

\section{LA POINTE (WISCONSIN)}

Nous nous servons du poste de La Pointe comme premier tremplin de recherche sur la société du bassin des Grands Lacs et du Saint-Laurent vivant de la traite des fourrures. Fondé en 1693, ce poste se trouve sur l'île Madeline, l'une des îles des Apôtres, juste au large de la péninsule de Chagouamigon [Chequamegon], au sud du lac Supérieur. La Pointe demeure une importante station de traite des fourrures et de pêche commerciale jusqu'au milieu du XIX ${ }^{\mathrm{e}}$ siècle. Elle a d'abord servi de poste français de traite des fourrures, de 1693 à 1759. En 1765, après la guerre de la Conquête, le poste a été réoccupé par des associés de Jean Baptiste Cadotte, commerçant de fourrures originaire de Bastican (Québec) établi à Sault-Sainte-Marie. Par la suite, son fils Michel Cadotte père a pris la relève et la direction du poste pour le compte des marchands de la Compagnie du Nord-Ouest (CNO), une entreprise montréalaise $e^{8}$.

Les mariages et les baptêmes de 1835 enregistrés à La Pointe au cours de la première année d'existence de la mission catholique de l'île Madeline

6. Bethel Saler et Carolyn Podruchny, «Glass Curtains and Storied Landscapes: The Fur Trade, National Boundaries, and Historians », dans Benjamin H. Johnson et Andrew R. Graybill, dir., Bridging National Borders in North America: Transnational and Comparative Histories (Durham, Duke University Press, 2010), p. 275-302.

7. Il existe deux études récentes au sujet du rôle dominant des Anichinabés dans l'histoire des Grands Lacs: Michael Witgen, An Infinity of Nations: How the Native New World Shaped Early North America (Philadelphie, University of Pennsylvania Press, 2012) et Michael A. McDonnell, Masters of Empire: Great Lakes Indians and the Making of America (New York, Hill and Wang, 2015).

8. John O. Holzhuter, Madeline Island \& the Chequamegon Region (Madison, WI, State Historical Society of Wisconsin, 1986), p. 24-25. Theresa M. Schenk, "The Cadottes: Five Generations of Fur Traders on Lake Superior ", dans Jennifer S. Brown, W. J. Eccles et Donald P. Heldman, dir., The Fur Trade Revisited: Selected Papers of the Sixth North American Fur Trade Conference (East Lansing, Michigan State University Press, 1994), p. 189-198. 
ont servi de portes d'entrée à notre analyse'. Bien qu'une partie de la population commerçante du lac Supérieur ait pu remplir ses obligations religieuses soit à la mission de Michilimackinac, soit dans les paroisses de la région de Montréal, la majorité ne l'avait manifestement pas fait. Les couples et les familles des diverses localités du lac Supérieur pratiquant la traite des fourrures se sont en effet rendus en masse à la mission pour recevoir les sacrements du mariage et du baptême, souvent pour régulariser ainsi des mariages à la façon du pays conclus depuis longtemps et légitimer, aux yeux de l'Église, les enfants issus de ces unions. Les registres de catholicité de 1835 offrent aux chercheurs une sorte de "capture d'écran" de la population canadienne-française et franco-anichinabée présente dans la région de l'ouest des Grands Lacs au début du XIX ${ }^{\mathrm{e}}$ siècle. À partir des noms inscrits dans les registres, nous avons procédé à une analyse des liens de filiation et de fraternité dans l'espoir de découvrir et de cartographier les réseaux sociaux existants au sein de la traite des fourrures dans la région des Grands Lacs. Cet exercice donne un premier aperçu de l'émergence de cette société lacustre très mobile, alimentée par les diktats du mercantilisme international et influencée par diverses priorités locales, régionales ou impériales; de plus, il donne aussi une idée des normes, des valeurs et des réseaux possibles qui ont contribué à la cimenter pendant plus d'un siècle.

\section{LA TRAITE DES FOURRURES DANS LA PARTIE OUEST DES GRANDS LACS}

Il subsiste dans les archives notariales, conservées par Bibliothèque et Archives nationales du Québec, plus de 21000 contrats d'engagement de voyageurs signés à Montréal entre 1714 et $1805^{10}$. À l'examen des destinations indiquées, il devient rapidement évident que la plupart des voyageurs s'engageaient pour travailler dans ce qu'on a appelé le commerce du Sud - la région de Michilimackinac, celle des Grands Lacs inférieurs

9. «Baptismal Records, 1835-1887, kept at La Pointe and Bayfield Indian missions by Iraneus Frederic Baraga, (part 1)", Lost in Canada? 16, 4 (été 1992), p. 183-195; Linda E. Bristol, comp., St. Joseph Mission and Holy Family Catholic Church Marriage Records, 1835-1880 (St. Paul, MN, Sump Press, 1994). Une seule sépulture a eu lieu au cours de la première année d'existence de la mission de La Pointe. Le 27 août 1835, Adélaïde, deux ans, fille de Michel Brissette et de Catherine Ogimakwe, a été inhumée dans le cimetière de la mission. L. E. Bristol, comp., Liber Defunctorum: St. Joseph Mission and Holy Family Catholic Church: Death Registry, 1835-1900 (Bayfield, WI, L.E. Bristol, 1994), p. 1.

10. Robert Englebert et Nicole St-Onge, «Paddling into History: French-Canadian Voyageurs and the Creation of a Fur Trade World, 1730-1804 ", dans Denis Combet, Luc Côté, et Gilles Lesage, dir., De Pierre-Esprit Radisson à Louis Riel : voyageurs et Métis/From Pierre-Esprit Radisson to Louis Riel: Voyageurs and Métis (Saint-Boniface, Presses universitaires de Saint-Boniface, 2014), p. 71-104. 


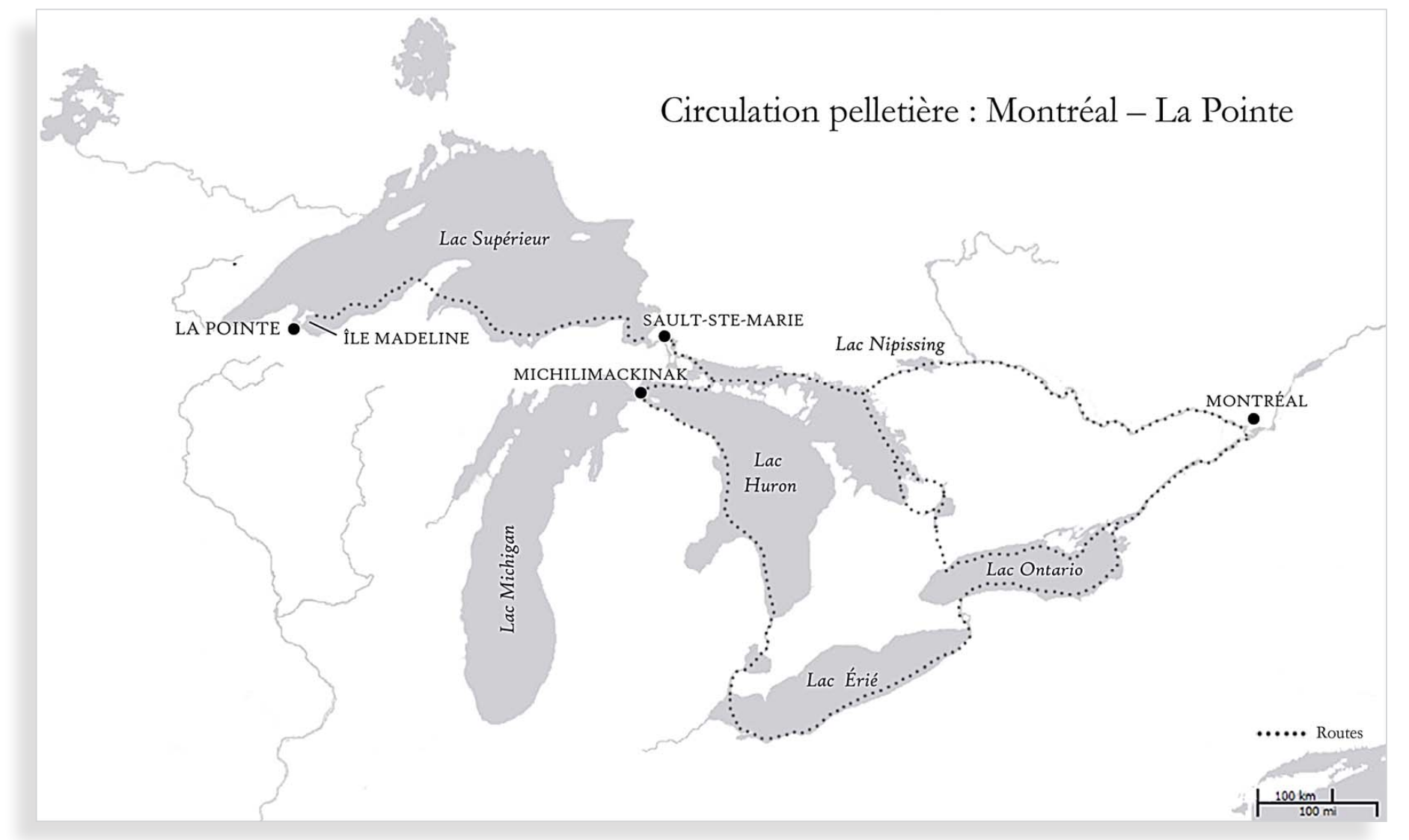


et le pays de l'Illinois. Avant 1805, ce commerce dépassait certainement en importance le commerce du Nord-Ouest, si ce n'est en ce qui concerne la quantité de fourrures récoltées, du moins quant au nombre de participants. Même après 1805, des hommes destinés au commerce du Sud continuent d'être embauchés à Montréal soit par de petites entreprises indépendantes établies à cet endroit, soit par la grande Michilimackinac Company (1806) ou la société qui lui a succédé, la South West Company (1811), et, dans sa dernière incarnation, l’American Fur Company (1817). Ce recrutement d'employés pour la traite des fourrures dans la région des Grands Lacs s'est poursuivi jusqu'aux années 1840. Ainsi, pendant plus d'un siècle y a-t-il eu circulation constante de personnes, de marchandises, de fourrures et d'information entre Montréal, l'ancienne métropole de la traite des fourrures, et le sud du bassin versant des Grands Lacs.

De 1817 jusqu'aux années 1840, l'AFC exploite le poste de La Pointe comme poste de traite des fourrures et de pêche commerciale. L'importance du poste dans les opérations de l'entreprise n'a cessé de croître, à tel point qu'en 1835, l'AFC déménage son centre administratif de Michilimackinac à l'île Madeline ${ }^{11}$. Attiré par la taille de la population associée à l'île et aux activités de l'entreprise à cet endroit, un prêtre missionnaire catholique, le père Frederic Baraga, ouvre une mission à La Pointe en $1835^{12}$.

\section{LA POPULATION DE LA POINTE}

Outre les registres de baptêmes, mariages et sépultures catholiques de 1835, les grands livres de l'AFC des années 1836-1842 pour La Pointe et ses postes satellites existent encore ${ }^{13}$. Ceux-ci font découvrir un intéressant éventail d'employés travaillant au sud et à l'ouest du lac Supérieur. Il s'agissait de jeunes hommes embauchés directement depuis Montréal; de "natifs du pays», comme on disait dans le bassin hydrographique de la baie d'Hudson, c'est-à-dire de fils ou de petits-fils du personnel de la traite des fourrures et de femmes locales; enfin, d'hommes au nom anichinabé qui sont souvent mentionnés comme travaillant dans la pêche commerciale de l'entreprise. Les noms des descendants de certaines des plus anciennes familles de marchands de fourrures du bassin des Grands Lacs

11. Harvey L. Carter, "Ramsay Crooks», dans Leroy R. Hafen, dir., The Mountain Men and the Fur Trade of the Far West (Glendale, CA, Arthur C. Clark Company, 1972), vol. 9, p. 131.

12. Chrysostom Verwyst, "Frederic Baraga», dans The Catholic Encyclopedia (New York, Robert Appleton Company, 1907), <www.newadvent.org/cathen/02282b.htm>.

13. New York Historical Society [NYHS], American Fur Company [AFC] Records 1831-1849, Manuscript Collection N10943345. Les grands livres associés à cette collection n'ont pas été microfilmés avec le reste de la collection; aussi faut-il les consulter sur place. 
et du fleuve Saint-Laurent - Cadotte, Nolin et Lorimier, par exemple sont facilement reconnaissables sur les listes d'employés. Ces livres de comptes aident à étoffer l'information trouvée dans les registres de catholicité à propos de la génération marquant le point d'entrée de cette analyse.

\section{ANALYSE DES REGISTRES DE MARIAGES ET DE BAPTÊMES DE LA POINTE POUR I835}

Le père Baraga a célébré 37 mariages entre le 2 août et le 25 décembre 1835 . Seulement cinq d'entre eux concernent des conjoints qui avaient manifestement l'un et l'autre des noms de famille anichinabés. Dans 13 autres cas, l'époux était soit un employé canadien-français de la traite des fourrures, soit le fils ou le petit-fils de l'un d'eux, tandis que l'épouse portait un patronyme anichinabé. Les 19 autres unions conjugales visaient des hommes et des femmes portant habituellement des noms de famille français; c'est dire qu'ils provenaient eux-mêmes de la vallée du Saint-Laurent ou que l'un de leurs ancêtres paternels en était originaire. Invariablement, les femmes portant des noms de famille français étaient filles ou petites-filles de commerçants ou de voyageurs canadiens-français et de femmes anichinabées. Au cours de ces cinq mêmes mois, Baraga a baptisé 186 individus, allant de nouveau-nés jusqu'à une personne ayant déclaré avoir 90 ans ${ }^{14}$. Quatrevingt-dix de ces personnes avaient des noms de famille anichinabés, tandis que les 96 autres portaient surtout des patronymes de voyageurs canadiensfrançais. En fonction de ce groupe initial de personnes, nous avons sélectionné pour une étude plus poussée les mariés ayant un nom de famille français et leur épouse anichinabée ou franco-anichinabée. Nous avons reconstitué les liens latéraux de parenté ou de parenté symbolique (p. ex., parrains et marraines, témoins au mariage, conjointe ou conjoint choisi) pour la population canadienne-française et franco-anichinabée qui se rassemblait à La Pointe en 1835. Puis, nous avons effectué une analyse en amont des liens familiaux de ces personnes afin de déterminer, dans la mesure permise par les sources, qui étaient leurs parents et grands-parents et quel était leur lieu de résidence; nous avons également tenté de reconstituer autant que possible leur parcours professionnel et leur histoire familiale. Un tel exercice contribue à déterminer la densité et la stabilité des liens de parenté à partir de 1835, dans la partie du bassin versant des Grands Lacs

14. Madeleine Kakigekwe, de La Pointe, a été baptisée à cet endroit le 18 octobre 1835 à l'âge de 90 ans. Son parrain a été Michel Cadotte père, et sa marraine, Madeleine Gwessewe (dite La Grue). «Baptismal Records, 1835-1887, kept at La Pointe and Baysfield Indian Missions», p. 183-192. 
et du Saint-Laurent où se pratiquait la traite des fourrures. Il aide aussi à remonter d'au moins deux générations et à avancer d'une génération, soit jusqu’à la période de la création des réserves au milieu du siècle.

\section{DES HISTOIRES ENTRELACÉES}

On trouve 50 noms de famille à prédominance canadienne-française concernant des personnes qui se marient ou qui leur servent de témoins dans les actes de mariage catholiques célébrés à La Pointe en 1835. À ces patronymes s'ajoutent les noms autochtones de 13 femmes anichinabées qui épousent des Canadiens français ou des Franco-Anichinabés. À première vue, ces personnes semblent constituer un groupe largement aléatoire et disparate. Toutefois, une analyse de réseau suivant les liens de parenté réelle ou symbolique révèle l'existence de relations entre la majorité d'entre eux; elle montre en outre la présence d'un regroupement supplémentaire en quatre groupes principaux. Autrement dit, la plupart des personnes présentes sur l'île Madeline en 1835 pour un baptême ou un mariage catholique avaient des liens réels ou symboliques (soit les parrains et les marraines, les témoins aux mariages, ou les liens par mariage) avec quatre familles fondées par des commerçants de fourrures canadiens-français et leurs épouses anichinabées. Selon un phénomène également constaté à Michilimackinac, sur l'île Mackinac, ces couples fondateurs et leurs descendants ont servi de points d'ancrage; ils ont maintenu ensemble des individus et des unités familiales très mobiles associés à la traite des fourrures qui vivaient et travaillaient dans la grande région située au sud du lac Supérieur ${ }^{15}$. Les quatre couples ayant ainsi servi de points d'ancrage sont: Augustin Bélanger et Josette Wabani; Pierre Côté et Angélique Chippewa; Louis Dufault et Marie Louise Medosky; Michel Cadotte père et Madeleine (La Grue) Equasayway.

\section{AUGUSTIN BÉLANGER ET JOSETTE WABANI}

Le Canadien français Augustin Bélanger, né vers 1770, signe en 1791 un contrat d'un an à Montréal avec la firme Todd, McGill \& Co. pour travailler comme équipier du milieu en canot "là où il sera envoyé». Mentionné par Michel Curot comme travaillant au poste de Yellow River de la CNO en 1803-1804, Bélanger est inscrit en 1804 comme employé de la division de Fond du Lac [aujourd'hui Duluth (Minnesota)] de la CNO,

15. Émilie Pigeon, «Réseaux sociaux catholiques et construction identitaire dans les Pays d'en haut: l'exemple du fort Michilimackinac (1741-1821)", Francophonies d'Amérique, 40-41 (2016), p. 83-112. 


\section{Représentation partielle de la famille Bélanger}

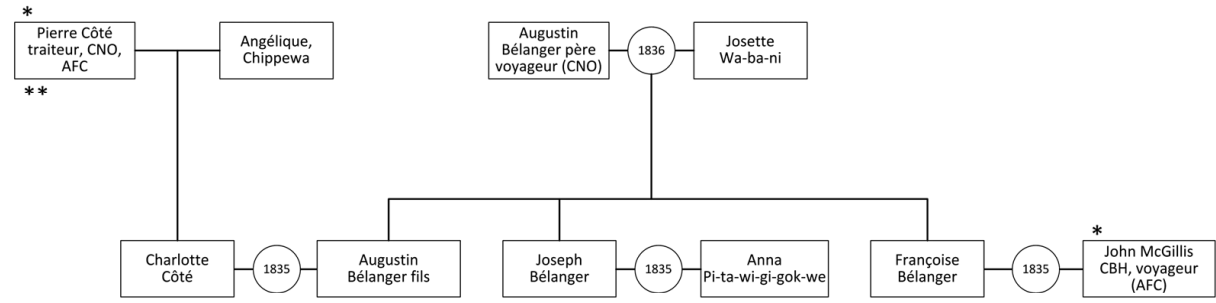

à l'extrémité sud du lac Supérieur ${ }^{16}$. De 1811 à 1816, Augustin continue de figurer dans les livres de la CNO comme employé de Fond du Lac. Sa fille Françoise voit le jour vers 1816. Dans sa demande de concession de terre (scrip) de 1839, celle-ci déclare être née dans le district du lac à la Pluie où son père était en poste ${ }^{17}$. En 1818 et jusqu'à la fusion, en 1821, de la $\mathrm{CNO}$ avec la Compagnie de la Baie d'Hudson (CBH), Augustin travaille à la division de la rivière Rouge de la $\mathrm{CNO}$, dans le bassin versant de la baie d'Hudson ${ }^{18}$.

À un moment donné entre 1821 et 1826, sa famille et lui retournent à l'est de la ligne laurentienne de partage des eaux. Un addendum à l'article $4 \mathrm{du}$ traité de 1826 avec les Chippewas, signé à Fond du Lac, promettait aux «sang-mêlé» de la tribu des lots de terre individuels «arpentés à l'ancienne façon française ${ }^{19}$ ». La liste des noms annexée au traité énumère ceux que la

16. Michel Curot, "A Wisconsin Fur-Trader's Journal, 1803-04», Collections of the State Historical Society of Wisconsin, 20 (1911), p. 396-471; et «Liste des bourgeois, commis, engagés et voyageurs de la Compagnie du Nord-Ouest, après la fusion de 1804 », dans L. R. Masson, Les Bourgeois de la Compagnie du Nord-Ouest (New York, Antiquarian Press, 1960 [1889-1890]), p. 395-413.

17. Theresa M. Schenck, All Our Relations: Chippewa Mixed Bloods and the Treaty of 1837 (Madison, WI, Amik Press, 2010), p. 94.

18. Provincial Archives of Manitoba (PAM), Hudson Bay Company Archives (HBCA), North West Company Account Books 1811-1821, F.4/32, fol. 99.

19. Selon le système de lots de rivière que l'on trouve habituellement dans la vallée du Saint-Laurent. 
tribu considérait comme admissibles à l'obtention de ces lots. Parmi eux figure Waubunequa, l'épouse d'Augustin Bélanger; ses enfants et elle devaient en effet recevoir chacun une de ces parcelles de terre ${ }^{20}$. Dix ans plus tard, à l'été 1836, Augustin Bélanger, «de La Pointe», épouse officiellement sa compagne de longue date, Josette Wabani, également «de La Pointe», à la mission catholique de l'île Madeline; il a 65 ans, elle en a 56. Josette Wabani/Waubunequa est plus tard décrite dans la demande de concession de terre d'un de ses fils en 1839 comme une "Chippewa du lac des Sables» [Big Sandy Lake (Minnesota)], région où un poste de traite de la CNO avait été établi en $1794^{21}$. Les témoins à la cérémonie nuptiale sont le commerçant canadien-français Jean Baptiste Corbin et le voyageur Alexis Charpentier. Ces deux hommes résidaient dans le district du lac Courte Oreilles et étaient mariés à des femmes de l'endroit ${ }^{22}$.

Augustin Bélanger père et Josette Wabani ont eu au moins trois enfants, qui ont tous été officiellement mariés par le père Baraga en 1835, un an avant le mariage de leurs parents. L’aîné des trois, Augustin fils, est né en 1805 au lac Yellow, mais l'affidavit accompagnant sa demande de concession de terre de 1839 indique qu'il a grandi principalement dans la région du lac des Sables, où vivait le peuple de sa mère. Augustin fils a travaillé pendant plusieurs années comme commerçant pour l'AFC au lac Winnibigoshish sur le haut Mississippi. En 1834-1835, Augustin fils est inscrit comme commerçant pour l'AFC dans le district de Fond du Lac ${ }^{23}$. En 1837, Augustin père et Augustin fils sont associés à l'équipe de l'AFC à l'île Royale en tant que pêcheurs commerciaux; ils sont engagés par la compagnie pour pêcher et vendre leurs prises à un prix prédéterminé ${ }^{24}$.

Au moment de la compilation de la liste des sang-mêlé en 1839, Augustin fils déclare de nouveau La Pointe comme lieu de résidence ${ }^{25}$. Il a pour

20. Charles J. Kappler, comp., Indian Affairs: Laws and Treaties (New York, AMS Press, 1971), 2, p. 268273, <https://dc.library.okstate.edu/digital/collection/kapplers/id/26109>. Ce traité n’a jamais été approuvé par le Sénat des États-Unis.

21. Irving Harlow Hart, "The Site of the Northwest Company Post on Sandy Lake», Minnesota History, 7, 4 (décembre 1926), p. 311-325.

22. Jean Baptiste Corbin a habité la majeure partie de sa vie dans la région du lac Courte Oreilles. Il y est mort en 1866 à l'âge de 88 ans, environ, et a été inhumé au cimetière de la réserve du lac Courte Oreilles. Ancestry.com. U.S., Find A Grave Index, 1600s-Current, <https://www.findagrave.com/ memorial/113685065>.

23. Theresa M. Schenck, dir., The Objibwe Journals of Edmund F. Fly, 1833-1849 (Lincoln, NE, University of Nebraska Press, 2012), p. 95.

24. NYHS, grands livres de l'American Fur Company pour 1837; et Grace Lee Nute, "The American Fur Company’s Fishing Enterprises on Lake Superior ", Mississippi Valley Historical Review, 12 (mars 1926), p. $483-503$.

25. James L. Hansen, «A List of Mixed-Blood Chippewa of Lake Superior, 1839», Lost in Canada?, 16,1 (printemps 1991), p. 31. 
épouse Charlotte Côté, née en 1809 à Fond du Lac. Elle est la fille de Pierre Côté, commerçant de la CNO et de l'AFC à Fond du Lac, et de son épouse anichinabée Angélique/Margaret, une Chippewa de la région de la Folle Avoine $^{26}$. Les témoins au mariage Bélanger-Côté en août 1835 sont Jean Baptiste Corbin et Pierre Côté, le père de la mariée. Mais le couple s'était marié à la façon du pays avant 1835, puisqu'il s'est rendu à Michilimackinac en 1831 pour faire baptiser deux fils à cette mission ${ }^{27}$. Au moment de l'établissement de la liste des sang-mêlé en 1839, 6 enfants âgés de 18 mois à 11 ans vivent avec eux. Le lieu de naissance de ces enfants indiqué dans les registres témoigne du degré élevé de mobilité qu’impliquait la vie d'employé de la traite des fourrures. Augustin Bélanger fils déclare dans son affidavit que ses enfants sont nés au lac des Sables, au lac Leech, au lac Winnibigoshish (Minnesota) et à Isle River ${ }^{28}$. Le deuxième enfant de Bélanger, Joseph, voit le jour au lac des Sables en 1813. En août 1835, il épouse Anna Pitawigigijigokwe du lac Courte Oreilles. Anna a été baptisée la veille des noces. En 1839, Joseph Bélanger affirme que sa famille et lui vivent à La Pointe. Les témoins à son mariage sont Michel Cadotte père et Jean Baptiste Corbin. Michel Cadotte père était marié à une Anichinabée de la rivière Sainte-Croix, fille du chef Grue Blanche. Peu de mentions de Joseph Bélanger ont survécu dans les archives de l'AFC et des missions. Il reçoit un certificat de sang-mêlé (scrip) en 1839, après la signature du traité avec les Chippewas du lac Supérieur en 1837. Son dernier enfant est baptisé à La Pointe en 1843. S'il a travaillé pour l'AFC pendant ces années, c'est par intermittence. En 1894, Joseph Bélanger vit dans la réserve de White Earth, au Minnesota, avec une de ses filles et certains de ses petits-enfants. Selon les recenseurs, son nom anichinabé est $\operatorname{Koggog}^{29}$.

La troisième Bélanger à se marier à La Pointe en août 1835 est Françoise, 19 ans. Elle naît au moment où son père est en poste dans le district du lac à la Pluie ${ }^{30}$. Devant nos témoins récurrents, Jean Baptiste Corbin et Pierre Côté, Françoise épouse un employé de la traite des fourrures de 29 ans, John McGillis, né à Saint-Eustache, au Québec ${ }^{31}$. Celui-ci est le

26. J. L. Hansen, «A List of Mixed-Blood Chippewa...», p. 34.

27. Digital Archives Database, Liber Baptizatorum, missionis Sancta Anna, Mackinac 1823, <http:// dadp.ok.ubc.ca/profile/124334> et <http://dadp.ok.ubc.ca/profile/124333>. Voir aussi T. M. Schenck, All Our Relations..., p. 22.

28. J. L. Hansen, "A List of Mixed-Blood Chippewa...», p. 31.

29. Ancestry.com, U.S., Indian Census Rolls, 1885-1940, White Earth Agency, bobine M595_651, p. 18, ligne 13.

30. J. L. Hansen, «A List of Mixed-Blood Chippewa...», p. 27-45.

31. Programme de recherche en démographie historique (PRDH), Jean Baptiste McGillis, 1806-12-27, fiche no $2186759,<$ https: / / www.prdh-igd.com>. 
neveu de Hugh McGillis, un actionnaire de la CNO responsable du district de Fond du Lac en $1806^{32}$. En 1829, John McGillis signe à Montréal un contrat de trois ans avec la CBH à titre d'équipier du milieu et de menuisier dans les pays sauvages ${ }^{33}$. De toute évidence, il ne tarde pas à se rendre au sud des Grands Lacs, où il travaille pour l'AFC. En 1834, il travaille pour l'AFC comme batelier dans le district de Fond du Lac ${ }^{34}$. Dans les listes des sang-mêlé de 1839, les deux jeunes filles du couple, Charlotte et Zoé, sont inscrites comme étant nées à «LW", c’est-à-dire au lac Winnibigoshish, au Minnesota, ou au lac Winnipeg, au Manitoba, un district de la CBH. Charlotte et Zoé sont jugées inadmissibles à une concession de terre en vertu des dispositions du traité de 1837 avec les Chippewas du lac Supérieur ${ }^{35}$. En 1850, John McGillis, Françoise Bélanger et six de leurs enfants vivent dans le district de Sauk Rapids, au Minnesota ${ }^{36}$. La profession de John dans le recensement de 1850 est toujours celle de charpen$\operatorname{tier}^{37}$. En 1890-1892, John McGillis, âgé de 85 ans, apparemment veuf, vit avec sa fille aînée Charlotte McGillis et les deux enfants de cette dernière, Eddy et William, ainsi qu'avec la femme de William, Susan Jourdain ${ }^{38}$. Ils sont alors inscrits comme faisant partie de la bande des Chippewas du Mississippi du lac Gull, bande rattachée à la division administrative de Consolidated White Earth ${ }^{39}$.

\section{PIERRE Côté ET ANGÉLIQUE CHIPPEWA}

Pierre Côté, de Montréal, est engagé par Parker, Gerard et Ogilvie au printemps 1799 pour travailler dans les «dépendances du Sud» pendant un an

32. W. Stewart Wallace, comp. et dir., Documents Relating to the North West Company (Toronto, The Champlain Society, 1934), p. 221.

33. Société historique de Saint-Boniface (SHSB), Base de données des engagements des voyageurs, Jean McGillis, 1829-01-21, <http://shsb.mb.ca/engagements_voyageurs >.

34. T. M. Schenck, All Our Relations..., p. 94.

35. J. L. Hansen, "A List of the Mixed-Blood Chippewa... », p. 42.

36. T. M. Schenck, All Our Relations..., p. 94.

37. Ancestry.com, 1850 United States Federal Census, Sauk Rapids, Benton, Minnesota Territory, bobine M432_367, p. 1A, image 6.

38. Susanne Jourdain est probablement la petite-fille de Joseph Jourdain, un voyageur et guide de la région de Montréal qui travaillait pour la CNO en 1804 dans le district du lac à la Pluie. En 1818, celui-ci signe un contrat avec lord Thomas Selkirk pour servir de guide de Montréal à la rivière Rouge. Dans un ajout au même contrat, Joseph Jourdain accepte de travailler comme chasseur durant un an à partir du 6 septembre 1818. Il s'établit à la rivière Rouge pendant cinq ans sur un terrain riverain que lui a concédé Selkirk. SHSB, Base de données des engagements des voyageurs, Joseph Jourdain, 1818-06-18, <http:// shsb.mb.ca/engagements_voyageurs $>$. Jourdain a eu quatre fils nés au lac Red qui ont tous réclamé des certificats de sang-mêlé à la suite du traité de 1836 avec les Chippewas du lac Supérieur. On le leur a refusé parce que leur mère était une «Muskego originaire du territoire de la baie d'Hudson». T. M. Schenck, All Our Relations..., p. 81.

39. Ancestry.com, U.S., Indian Census Rolls, 1885-1940, White Earth Agency, M595_650, p. 1, ligne 18. 
comme équipier du milieu en canot et charpentier. Le 30 juillet 1806, il est inscrit comme interprète dans le district de Fond du Lac de la $\mathrm{CNO}^{40}$. Entre 1812 et 1816, les livres de comptes de la CNO qui subsistent révèlent qu'il continue à travailler dans la région de Fond du Lac; il gagne alors un salaire de 600 livres ancien cours et son compte est débité chaque année pour divers articles ${ }^{41}$. Selon l'ethnohistorien Bruce White, Côté est engagé par la South West Company de 1816 à 1817. En 1818, l'AFC l'emploie à Fond du Lac, d'abord comme interprète pour 2400 livres, puis, l'année suivante, comme marchand ${ }^{42}$. En 1834-1835, Côté est encore inscrit comme interprète de l'AFC à Fond du Lac ${ }^{43}$. En 1836, il est responsable des pêches commerciales à partir du poste de Grand Portage. La première épouse de Pierre Côté, Angélique Chippewa, venait probablement de la région du lac des Sables ${ }^{44}$. Elle mourut quelque temps avant les négociations du traité de 1826, mais on promit à chacun de ses enfants une section de terre de 640 acres à titre de "sang-mêlé de la tribu »" En 1830, à la mission de Michilimackinac, Côté se remarie à Margaret Roussain, fille d'Eustache Roussain, commis de la $\mathrm{CNO}$ et de l'AFC à Fond du Lac, et d'une Chippewa de la région de la Folle Avoine. Margaret, ses frères et ses sœurs se sont également vu promettre des terres par le traité de Fond du Lac de $1826^{46}$.

Pierre Côté a eu au moins deux enfants avec sa première femme: une fille, Charlotte Côté, susmentionnée, mariée à Augustin Bélanger fils, et un fils, Henri Côté, né en 1813 à Fond du Lac. Le 9 septembre 1835, à la mission de La Pointe, Henri épouse Isabelle Gournon, 20 ans, elle aussi de Fond du Lac. Joseph Montreuil fils et Michel Cadotte fils sont témoins. Joseph Montreuil fils, interprète de l'AFC à Fond du Lac, était marié à Louise Dufault, une Franco-Anichinabée, qui était la cousine paternelle de la mariée. Ces deux femmes étaient les petites-filles d'un chef héréditaire du lac du Flambeau. Pour sa part, Michel Cadotte fils, commerçant de l'AFC, était marié à l'Anichinabée Ester Ossinagjeeunoqua, du lac du Flambeau, la fille du chef Kishkiman dit Homme Fier ${ }^{47}$. Les témoins aux cérémonies de mariage sont presque toujours des hommes bien branchés,

40. W. S. Wallace, Documents Relating to the North West Company, p. 221.

41. HBCA, Livres de comptes de la CNO, F.4/32, 218.

42. Bruce M. White, The Fur Trade in Minnesota: An Introductory Guide to Manuscript Sources (St. Paul, MN, Minnesota Historical Society Press, 1977), p. 39.

43. T. M. Schenck, The Objibwe Journals of Edmund F. Fly..., p. 461.

44. L'affidavit de 1839 accompagnant la demande de concession de terre du fils Côté, Henri, est reproduit dans T. M. Schenck, All Our Relations..., p. 52.

45. C. J. Kappler, Indian Affairs: Laws and Treaties, 2, p. 268-273.

46. C. J. Kappler, Indian Affairs: Laws and Treaties, 2, p. 268-273.

47. T. M. Schenck, All Our Relations..., p. 52. 


\section{Représentation partielle de la famille Cadotte}

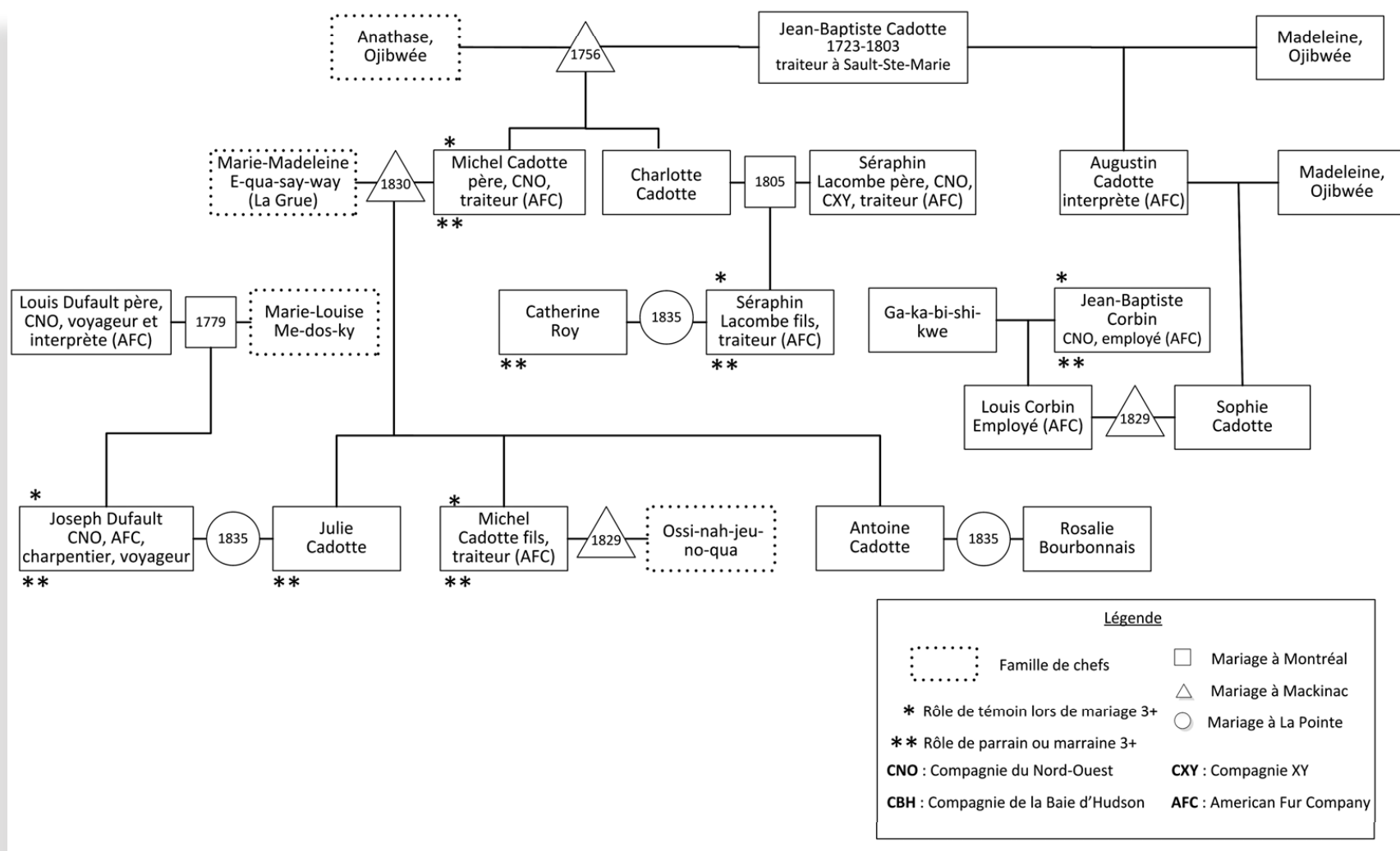


tant dans le monde franco-catholique de la traite des fourrures que dans les communautés anichinabées des Grands Lacs. Selon les affidavits de 1839 concernant les sang-mêlé chippewas, Isabelle Gournon est née dans la région de Sault-Sainte-Marie. À cette date, Henri Côté et Isabelle Gournon ont deux jeunes enfants : l'aîné, né à Fond du Lac, et le cadet, à Grand Portage. En 1834-1835, Henri est marchand pour l'AFC à Fond du Lac. En 1839, il est autorisé à faire du commerce dans le district de Grand Portage où vivent son père et sa belle-mère. En 1847, Henri et sa famille immédiate sont rendus dans la région de Sault-Sainte-Marie. En 1898, l'un des fils d'Henri et d'Isabelle, William Lawrence, épouse Julie Amyotte, fille d'Amable Amyotte et de Julie Nolin, à la mission voisine de Garden River $^{48}$. La mère d’Isabelle Gournon-Côté est Angélique Dufault, la fille du couple suivant dont il sera ici question.

\section{LOUIS DUFAULT ET MARIE LOUISE MEDOSKY}

Dans les actes de mariage de La Pointe de 1835, les descendants de Louis Dufault père, voyageur canadien-français, et de son épouse Marie Louise Medosky, attirent l'attention. Voyageur né à Longueuil, au Québec, en 1747, Louis Dufault père signe plusieurs contrats à Montréal concernant des destinations dans la région de Michilimackinac ${ }^{49}$. Lors d'un de ses séjours dans les Pays d'en Haut, Louis Dufault contracte un mariage à la façon du pays avec une Anichinabée du lac du Flambeau, fille d'un chef héréditaire local appelé Aile de Canard. En 1777, à la fin de son contrat, Dufault plie bagage avec sa femme et ses deux jeunes filles et retourne dans la vallée du Saint-Laurent. Son épouse, que l'on dit être Marie Louise Mentosaky, de la "nation saulteuse ", et ses enfants ne tardent pas à y être baptisés. En 1779, le couple se marie officiellement à l'église paroissiale de Pointe-Olivier [Saint-Mathias], au Québec, et au cours de la même cérémonie, il reconnaît et légitime ses deux filles et son fils ${ }^{50}$.

Le couple demeure dans la région de Longueuil jusqu'en 1784, date à laquelle Louis Dufault signe un contrat pour la traite des fourrures avec John Gregory \& Co. pour être second de devant dans un canot puis interprète à Michilimackinac. Sa femme l'accompagne dans la région des Grands Lacs, avec seulement quelques-uns de ses enfants, semble-t-il. À partir de

48. Digital Archives Database Project (DAD), William Lawrence Côté et Julia Amyotte, 1898/03/14, $<$ http://dadp.ok.ubc.ca/profile/327031>.

49. SHSB, Base de données des engagements des voyageurs, Louis Dufault, diverses dates, $<$ http:// shsb.mb.ca/en/Voyageurs_database>.

50. Programme de recherche en démographie historique (PRDH), Dufault-Mentosaky 1779-02-15, fiche $\mathrm{n}^{\circ} 247865,<$ http: / / www.prdh-igd.com>. 


\section{Représentation partielle de la famille Côté}

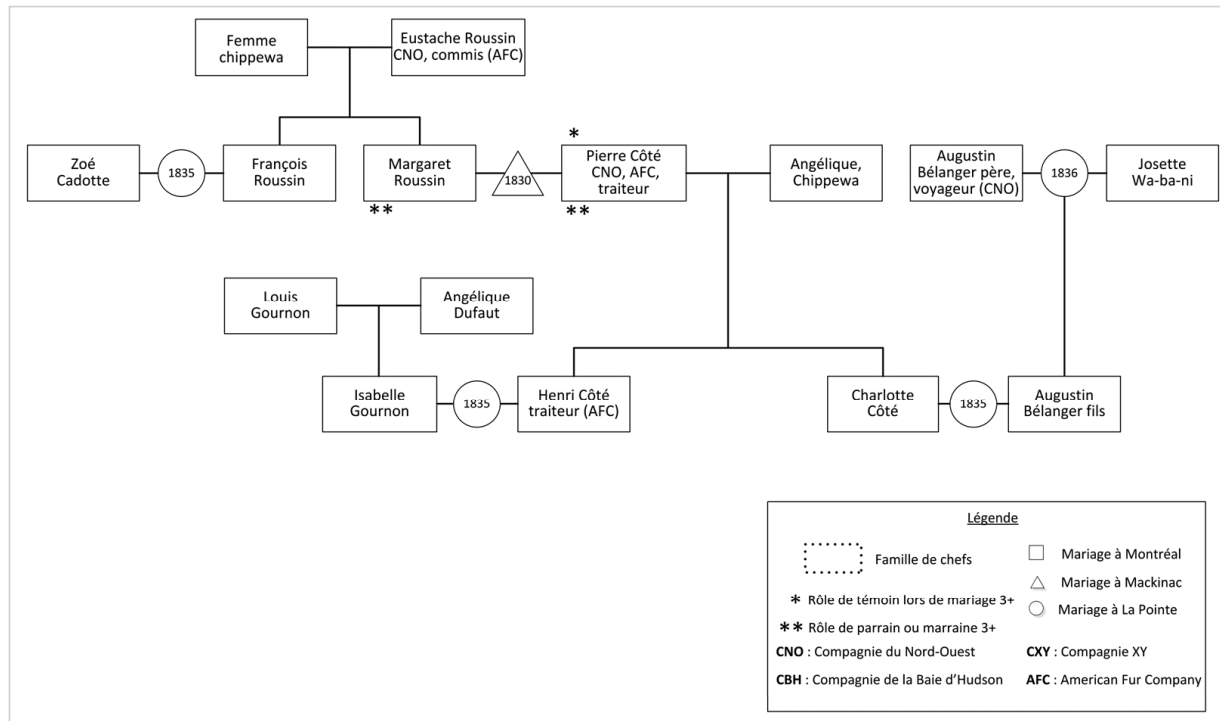

cette date, Louis Dufault est cité dans divers récits, dont les mémoires publiés par Jean Baptiste Perrault ${ }^{51}$. Ce dernier déclare avoir rencontré Dufault en 1791, au poste du lac du Flambeau de la CNO, où le père de Marie Louise Mentosaky était chef et où au moins deux garçons Dufault sont nés, Louis fils et Joseph. À la fin de sa carrière, Louis Dufault père, comme d'autres employés de longue date de la traite des fourrures, choisit de s'établir à Sault-Sainte-Marie, où il occupe le lot $n^{\circ} 12$ sur la rivière. Après sa mort en 1817, ses fils vendent la propriété à Antoine Lalonet avec l’aide de membres de la famille Cadotte ${ }^{52}$.

Louis Dufault fils, né vers 1781, travaille pour la CNO jusqu'en 1816 comme interprète au poste de l'entreprise à Fond du $\mathrm{Lac}^{53}$. Puis, en 1817, c'est l'agent de l'AFC à Michilimackinac, Ramsay Crooks, qui l'embauche pour travailler dans la région de Fond du Lac ${ }^{54}$. Louis Dufault fils et son

51. Jean Baptiste Perrault et J. S. Fox, «Narrative of the Travels and Adventures of a Merchant Voyageur in the Savage Territories of Northern America Leaving Montréal the 28th of May 1783 (to 1820)", Historical Collections and Researches Made by the Michigan Pioneer and Historical Society, 37 (1910), p. 508-619.

52. John P. DuLong, «Pauwayteeg Public Land Claims, Sault Ste. Marie, Michigan Territory, 1823 », dans The Cadottes: A Métis Fur Trade Family of Lake Superior (2018), <www.habitant.org/cadotte/index. htm $>$.

53. HBCA, grands livres de la CNO, F.2/32, 309.

54. Donna Valley Russell, dir. et comp., Michigan Voyageurs from the Notary Book of Samuel Abbott, Mackinac Island 1807-1817 (Detroit, Detroit Society for Genealogical Research, 1982). Nombre d'employés 
frère cadet Joseph sont inscrits comme employés de la compagnie dans un registre des employés de l'AFC de 1818-1819 conservé au siège social de l'entreprise, à Michilimackinac. Louis fils est toujours interprète à Fond $\mathrm{du}$ Lac, tandis que Joseph travaille comme batelier à Sault-Sainte-Marie, mais sa destination est le lac du Flambeau; il s'agit de la région natale de Joseph, un coin de pays où résidaient encore de nombreux parents de sa mère ${ }^{55}$.

Joseph Dufault est l'époux du premier couple que marie le père Baraga à La Pointe. Le 2 août 1835, Joseph Dufault, résident de La Pointe, reçoit le sacrement du baptême et légitime son union avec Julie Cadotte, 37 ans, résidente de La Pointe, fille de Michel Cadotte père et de Marie Madeleine («La Grue») Equaysayway ${ }^{56}$. Michel Cadotte père et Madeleine La Grue avaient officialisé leur propre mariage à la façon du pays cinq ans auparavant, à Michilimackinac. Les témoins de la cérémonie à La Pointe sont Alexis Charpentier et Pierre Côté, des hommes dont les noms sont souvent mentionnés. Au milieu des années 1830, Joseph Dufault travaille comme charpentier à La Pointe; il gagne 350 dollars par année et reçoit une double ration $^{57}$. Joseph et Julie (Cadotte) Dufault, ainsi que leur fils Michel, âgé de 9 ans, sont inscrits sur la liste de 1839 des sang-mêlé chippewas du lac Supérieur comme étant admissibles à une concession de terre. Néanmoins, en 1890, Michel Dufault fils et son épouse Josette Roy sont membres à part entière de la bande des Chippewas de Fond du Lac. Quatre ans plus tard, le même couple est inscrit comme membre ojibwé de la bande de la division administrative de La Pointe. Finalement, quelque temps après cette date, cette branche de la famille déménage dans la réserve de White Earth, où Michel Dufault meurt et est enterré en $1916^{58}$.

Le frère de Joseph Dufault, Louis fils, officialise également son mariage à la façon du pays quatre mois après son frère. Louis fils, 54 ans, est alors marchand pour l'AFC et responsable du poste du lac Red. Le journal d’Edmund Ely signale la présence de Louis fils au lac Red à trois reprises

de la CNO sont passés à l'AFC après 1816, la CNO limitant de plus en plus ses activités au territoire de l'Amérique du Nord britannique.

55. "American Fur Company Employees, 1818-1819», Collections of the State Historical Society of Wisconsin, vol. 12 (1892), p. 154-169.

56. L'identification de ses parents ojibwés est clarifiée dans T. M. Schenck, All Our Relations..., p. 35 et 66 .

57. NYHS, grands livres de l'AFC, 1837, La Pointe.

58. Ancestry.com, U.S., Indian Census Rolls, 1885-1940, M595_235, ligne 14; Schenck, All Our Relations..., p. 63-64. 
en 1833-1834 ${ }^{59}$. En se mariant le 25 décembre 1835, Louis fils suit l'exemple de son frère, de sa fille Louise (qui épouse le Franco-Anichinabé Joseph Montreuil fils) et de sa nièce Isabelle Gournon (qui épouse le FrancoAnichinabé Henri Côté), lesquels se marient eux aussi en 1835. L'épouse de Louis Dufault fils est Marie Onichi, peut-être une Anichinabée de La Pointe, baptisée, comme beaucoup d'autres, le jour de son mariage. Le frère de Louis, Joseph Dufault, et le voyageur canadien-français Louis Letendre en sont témoins ${ }^{60}$. Selon la liste des sang-mêlé chippewas de 1839 , le couple a encore cinq enfants vivant avec lui; comme ils sont nés au lac Leech, au lac des Sables, au lac Red ou à La Pointe, ils sont donc admissibles à une concession de terre au lac Supérieur.

Comme nous l'avons indiqué précédemment, Louise, la fille de Louis Dufault et de Marie Medosky, épouse Joseph Montreuil, natif du lac des Sables. Celui-ci est le fils d'un voyageur canadien-français de la CNO, Joseph Montreuil (dit Sedilot) père, qui a quitté Montréal en 1791 pour travailler dans des postes non précisés de la CNO dans la région du lac Supérieur. En 1804, Joseph père travaillait encore pour la CNO, au poste de la rivière Chippewa ${ }^{61}$. En 1822, il besognait pour l'AFC dans le district du Mississippi supérieur. Lorsque son fils Joseph, né d'une Anichinabée du lac Red Cedar au nom inconnu, épouse Louise Dufault à La Pointe, les témoins sont, comme d'habitude, Michel Cadotte fils et Alexis Charpentier, interprète canadien-français de l'AFC au lac Courte Oreilles ${ }^{62}$.

\section{MICHEL CADOTTE PÈRE ET EQUASAYWAY}

Michel Cadotte père est le fils d'un commerçant de fourrures de SaultSainte-Marie et de La Pointe, Jean Baptiste Cadotte. Sa mère est probablement Marie Anastasie Nipissing, une Ojibwée du clan Awause (le clan du Poisson-Chat) de Sault-Sainte-Marie que Jean Baptiste épousa officiellement à la mission de Michilimackinac en $1756^{63}$. À un moment donné après cette

59. T. M. Schenck, The Ojibwe Journals of Edmund E. Ely..., p. 79; «American Fur Company Invoices, 1821-22», Collections of the State Historical Society of Wisconsin, 11 (1888), p. 370 et suiv.

60. SHSB, Base de données des engagements des voyageurs, Louis Letendre, 1828-02-05, <http:// shsb.mb.ca/en/Voyageurs_database>. Fils d'un journalier de Sorel, au Québec, Louis Letendre a été embauché par l'AFC en 1828 par voie de contrat de trois ans. Dans le recensement de 1870 pour Green Bay, au Wisconsin, le Canadien Louis Letendre, âgé de 65 ans, est décrit comme célibataire et ouvrier. Ancestry.com, 1870 United States Federal Census.

61. L. R. Masson, «Liste des bourgeois, commis, engagés et voyageurs de la Compagnie du NordOuest...», p. 395-413.

62. NYHS, grands livres de l'AFC, groupe du lac Courte Oreilles, grand livre de 1837.

63. Joseph Tassé, "Memoir of Charles de Langlade», Collections of the State Historical Society of Wisconsin, 7 (1876), p. 157-160; Alexander Henry, Travels and Adventures in Canada and the Indian Territories between the Years 1760 and 1776 (Boston, Little Brown and Company, 1901), p. 96-122. 
cérémonie, le couple séjourne à Montréal, où Marie Anastasie meurt et fait l'objet d'une sépulture catholique en $1776^{64}$. Comme nous l'avons mentionné, l'épouse de Michel Cadotte père est Madeleine («La Grue») Equasayway, du grand clan Ajijaakwe (le clan de la Grue). Madeleine, une femme éminente, est la fille d'un chef de la rivière Sainte-Croix, Grue Blanche, qui déménagea avec sa bande dans la région de Chagouamigon; elle est également la sœur d'un chef de la région de La Pointe, Gagaganah ${ }^{65}$. Michel et Madeleine officialisent eux aussi leur union à Michilimackinac à l'été 1830. Au cours de cette même cérémonie, Michel et Madeleine légitiment leurs sept enfants, entre autres Julie Cadotte-Dufault dont il a été question ci-dessus ${ }^{66}$. Le premier témoin de cette cérémonie à Michilimackinac est Alexis Corbin, fils de Jean Baptiste Corbin, le directeur du poste de Michel Cadotte au lac Courte Oreilles, et de son épouse anichinabée originaire du lac Courte Oreilles, Gagabishikwe. Le deuxième témoin est William McGulpin, boulanger et agriculteur résidant à Michilimackinac depuis 1817 et conjoint de Madeleine Bourassa, membre d'une importante famille mackinac d'origine franco-odawa dont les membres sont très visibles dans les registres de catholicité de Michilimackinac ${ }^{67}$. Par le traité de 1826 avec les Chippewas négocié à Fond du Lac, Michel Cadotte père, Equaysayway et chacun de leurs enfants "vivant aux États-Unis» se voient promettre une section de terres «arpentées à l'ancienne façon française» le long de la rivière Sainte-Marie ${ }^{68}$.

Comme ses autres frères, Michel Cadotte père a séjourné dans sa jeunesse à Montréal, où il a vécu chez la parenté de son père et reçu une éducation. Dans les années 1780, il retourne dans la région des Grands Lacs et s’associe alors à la CNO. À partir de 1817, ce marchand collabore de façon intermittente avec l'AFC dans les régions de la pointe Chagouamigon, de la rivière Chippewa, du lac Courte Oreilles et de la rivière Sainte-Croix. Quelque temps avant 1804, il choisit de s'établir au poste de La Pointe, sur l'île Madeline, un endroit commercialement stratégique que de nombreux

64. DAD, Jean Baptiste Cadotte-Marie Anastasie Neophite, 1756-10-28, <http://dadp.ok.ubc.ca/ profile/325726> ; T. M. Schenck, All Our Relations..., p. 35 ; PRDH, Marie Anastasie Algonquienne, tribu des Népissingues, 1776-05-18, fiche $\mathrm{n}^{\circ}$ 82203, <https:/ / www.prdh-igd.com>.

65. Brenda J. Child, Holding Our World Together: Ojibwe Women and the Survival of Community (New York, Penguin Books, 2012), p. 31-62.

66. DAD, Michel Cadotte-Marie Madeleine La Grue, 1830-07-26, <http://dadp.ok.ubc.ca/ profile/325814>.

67. Susan Sleeper-Smith, "Women, Kin, and Catholicism: New Perspectives on the Fur Trade», dans Susan Sleeper-Smith, dir., Rethinking the Fur Trade: Cultural Exchanges in an Atlantic World (Lincoln, NE, University of Nebraska Press, 2009), p. 457.

68. C. J. Kappler, Indian Affairs: Laws and Treaties, 2, p. 268-273. 


\section{Représentation partielle de la famille Dufault}

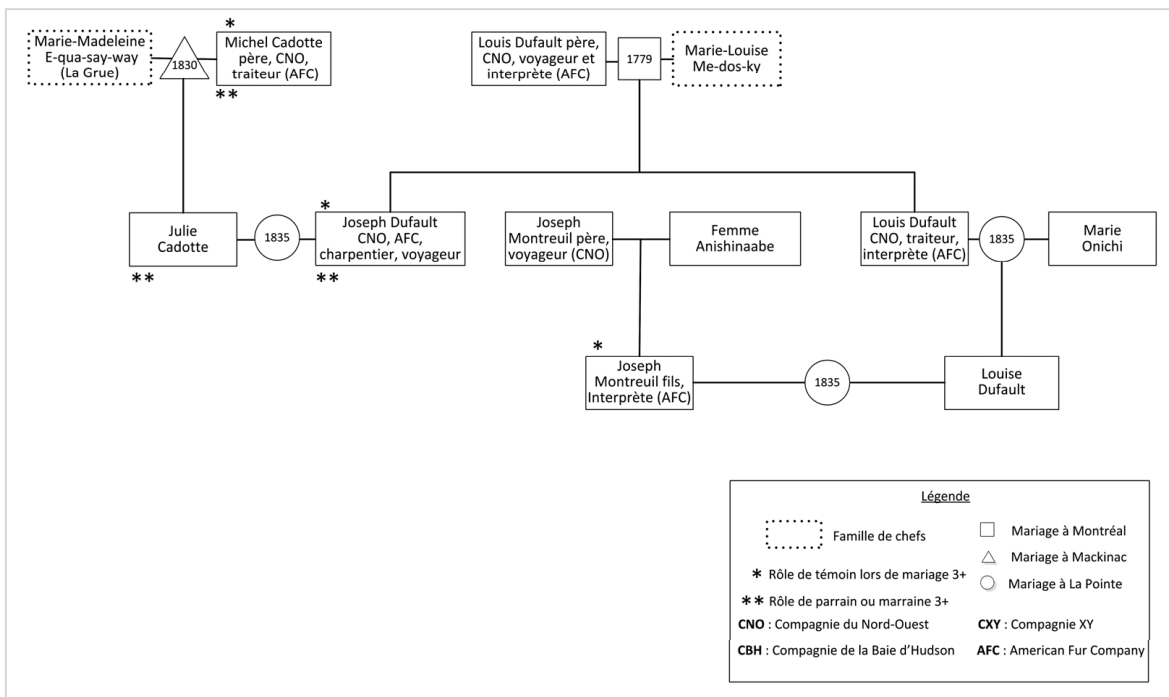

Anichinabés du lac Supérieur considéraient en outre d'une grande importance spirituelle. Sa femme et lui ont plusieurs parents parmi les dirigeants anichinabés de l'île Madeline et la péninsule de Chagouamigon en général ${ }^{69}$. Le poste de La Pointe devient la principale base d'opérations de Michel Cadotte père $^{70}$. Comme plusieurs autres commerçants de fourrures du lac Supérieur, Michel Cadotte père achète la citoyenneté américaine à l'été 1820 pour s'assurer de pouvoir poursuivre sans entrave ses activités de traite des fourrures au sud de la frontière ${ }^{71}$. Michel Cadotte père demeure sur l'île jusqu'à sa mort en 1837. Il est inhumé à La Pointe, au cimetière de la mission $^{72}$.

D'autres membres de la famille Cadotte s'établissent eux aussi au sud du lac Supérieur. La sœur de Michel père, Charlotte Cadotte, est baptisée et se marie en 1805 lors d'une cérémonie paroissiale à L'Assomption, au Québec; elle officialise ainsi son mariage à la façon du pays avec François Séraphin Trullier dit Lacombe (aussi connu sous le nom de Séraphin

69. Jane C. Bush, People and Places: A Human History of the Apostle Islands (Omaha, NE, US National Park Services, 2008), p. 35-37.

70. "A Wisconsin Fur-Trader's Journal, 1804-05 ", Collections of the State Historical Society of Wisconsin, 19 (1910), p. 175.

71. T. M. Schenck, All Our Relations..., p. 35.

72. L. E. Bristol, Liber Defuntorum, 2, inscription du 8 juillet 1837. 
Lacombe), un marchand canadien-français du lac à la Pluie ${ }^{73}$. Leurs trois enfants nés au lac à la Pluie, dont Séraphin fils, âgé de deux ans, sont légitimés au cours de la même cérémonie. Séraphin fils fait ses études à L'Assomption, où ses parents sont installés. À l'âge de 15 ans, peu après le décès de son père en 1817, il retourne dans les Pays d'en Haut et s'adonne au commerce dans les régions du lac à la Pluie, du lac Leech et du lac des Bois. En 1834, il travaille comme interprète de l'AFC à Fond du Lac auprès de son oncle maternel Michel Cadotte père et de divers cousins. En 1835, à la mission de La Pointe, il épouse Catherine Roy, une Franco-Anichinabée. Dans sa demande de certificat de sang-mêlé en 1839, Séraphin déclare être apparenté à Bresche, ou Dent Cassée, un chef anichinabé du clan du Huard du lac des Sables ${ }^{74}$. Séraphin Lacombe fils meurt en décembre 1840 et est inhumé à La Pointe ${ }^{75}$.

Les nièces de Michel Cadotte père, Sophie et Zoé, se marient toutes deux à des commerçants de fourrures ${ }^{76}$. En 1829, à Michilimackinac, Sophie épouse Louis Corbin, batelier de l'AFC au lac Courte Oreilles et fils du commis Jean Baptiste Corbin et de Gagabishikwe, une Anichinabée du lac Courte Oreilles. En 1835, à La Pointe, Zoé Cadotte épouse officiellement François Roussain, 20 ans, fils d'Eustache Roussain, un important marchand canadien-français de Fond du Lac lié à la CNO et à l'AFC, et d'une Anichinabée de nom inconnu de la région de la Folle Avoine ${ }^{77}$.

Lors d'une cérémonie à Michilimackinac en 1829, Michel Cadotte fils épouse celle qui était sa femme à la façon du pays, Ossinahjeeunoqua, fille d'un chef respecté du lac du Flambeau. Comme c'est le cas de nombreuses personnes dont il est question dans cet article, Ossinahjeeunoqua et ses enfants se voient promettre des concessions de terres destinées aux sangmêlé dans le traité de 1826 avec les Chippewas de Fond du Lac. Avec ce mariage, Michel Cadotte fils est lié non seulement à une famille canadienne-française influente de la vallée du Saint-Laurent engagée dans le commerce des fourrures, mais aussi, par l'entremise de sa grand-mère,

73. John P. DuLong, "Charlote Cadotte: The "Vivacious" Wife of the Fur Trader Séraphin Lacombe», Michigan's Habitant Heritage, 36, 3 (juillet 2015), p. 101-116.

74. T. M. Schenck, All Our Relations..., p. 82.

75. John P. DuLong, "Jean Baptiste Cadotte’s Second Family: Genealogical Summary-Part 2», Michigan's Habitant Heritage, 37, 1 (janvier 2016), p. 49-50.

76. Sophie et Zoé étaient les filles d'Augustin, le demi-frère de Michel, et de son épouse Madeleine, une Ojibwée.

77. On connaît trois femmes anichinabées à Eustache Roussain: Shauwunaubunoqua, Wauwaussumoqua et Payshaubunoqua. Leurs enfants se sont tous vu offrir des terres en concession dans le traité non ratifié avec les Chippewas de 1826. 
de sa mère et de son épouse, à de multiples familles anichinabées du lac Supérieur, des familles influentes.

L'autre fils de Michel Cadotte père, Antoine Cadotte, est baptisé et se marie le 13 septembre 1835. Sa cérémonie à La Pointe officialise son union à la façon du pays avec Rosalie Bourbonnais, peut-être fille d'Amable Bourbonnais, un voyageur canadien-français de la région de Vaudreuil, au Québec, qui a été embauché à Montréal en 1802 pour travailler dans le «Nord-Ouest " $^{78}$. De 1811 à 1821, Amable Bourbonnais est stationné à Fort William, où Rosalie, "à demi-Chippewa", voit le jour vers 1810 d'après la demande de certificat de sang-mêlé formulée par Antoine Cadotte en 1839 pour ses enfants ${ }^{79}$.

\section{LES LIENS DE PARENTÉ SYMBOLIQUES}

La formalisation d'alliances par le biais de liens de parenté spirituelle catholiques constitue une autre activité de réseautage à La Pointe en 1835 . Par le parrainage et la présence comme témoins au mariage, les individus et les familles créent et rendent publics des liens d'obligations et de devoirs les uns envers les autres et envers la génération suivante née dans le monde de la traite des fourrures. Ces liens symboliques viennent cimenter ceux acquis par les Canadiens français ou les Anichinabés en matière de parenté familiale ou matrimoniale ou s'ajoutent à ceux-ci.

Le réseau de parenté symbolique de La Pointe en 1835 est principalement composé des descendants de Jean Baptiste Cadotte, marchand de fourrures du XVIII ${ }^{\mathrm{e}}$ siècle à Sault-Sainte-Marie, et de ses deux épouses successives, Marie Anasthasie et Madeleine, deux Ojibwées. Plusieurs de ses fils, de ses filles et de leurs conjoints, ainsi que les petits-enfants et leurs conjoints, sont présents à La Pointe, à titre de parrains, de marraines ou de témoins. L'attrait de ces personnes liées à Cadotte peut être double. Beaucoup d'hommes et de femmes de la famille Cadotte étaient instruits, et les femmes ont certainement épousé d'importants marchands de fourrures du lac Supérieur. Par exemple, Sophie Cadotte a épousé Louis Corbin, marchand pour l'AFC au lac Courte Oreilles, tandis que Zoé Cadotte a épousé François Roussain, marchand à Fond du Lac. La sœur de François, Margaret Roussain, et son mari, Pierre Côté, ont joué un rôle prépondérant comme parrain et marraine et comme témoins à des mariages. Charlotte Cadotte a épousé Séraphin Lacombe, un important commerçant du lac à la Pluie;

78. SHSB, Base de données des engagements des voyageurs, Amable Bourbonnais, 1802-03-09, $<$ http://shsb.mb.ca/engagements_voyageurs $>$.

79. HBCA, grands livres de la CNO, 1811-1821, F.4/32, fol. 114, Amable Bourbonnais. 


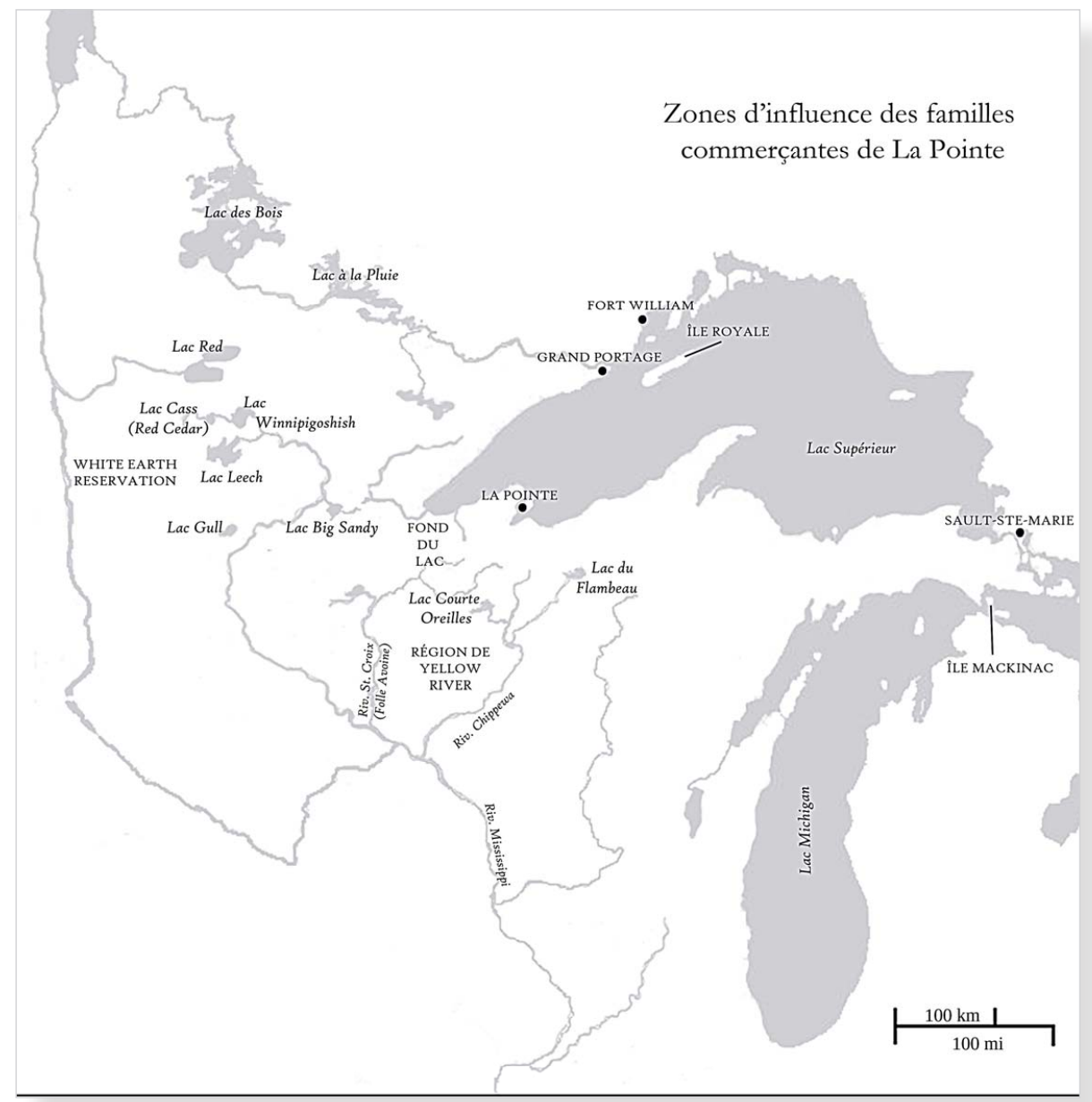

leur fils et leur belle-fille sont également très visibles comme parrain et marraine et comme témoins à des mariages ${ }^{80}$.

Les réseaux parallèles des familles alliées au sein des Anichinabés des Grands Lacs - des familles de chefs et des clans - se tissaient à l'intérieur de ces cérémonies de formation d'alliances catholiques canadiennes-françaises. Toutes les familles et tous les individus de La Pointe visibles dans les registres de catholicité de 1835 avaient apparemment des liens avec des

80. John P. DuLong, "Jean Baptiste Cadotte’s Second Family, Genealogical Summary», Parts 1-3, Michigan's Habitant Heritage, 36, 4 (octobre 2015), p. 188-198; 37, 1 (janvier 2016), p. 43-56; 37, 2 (avril 2016), p. 85-97; 37, 3 (juillet 2016), p. 156-168. 
membres importants de la population anichinabée environnante ${ }^{81}$. La mère de Michel Cadotte père est issue d'un clan de Poissons-Chats bien branché, une famille de chefs de la région de Sault-Sainte-Marie. Son épouse, Madeleine La Grue, du clan dominant de la Grue, était quant à elle fille d'un chef héréditaire de la Folle Avoine. En outre, le frère de Madeleine était chef dans la région de La Pointe lorsque Cadotte a établi son poste de traite sur l'île, ce qui a été très utile au marchand de fourrures qu'était Michel. Michel Cadotte fils a pour sa part épousé la fille d'un chef du lac du Flambeau, et sa fille Julie a épousé Joseph Dufault, menuisier et interprète pour l'AFC au lac du Flambeau, dont la mère, Marie Louise Medosky, était la fille du chef Aile de Canard. Ainsi, les personnes qui ont assisté à des baptêmes et à des mariages et qui ont assumé pour la vie les obligations de parrain ou de marraine n'étaient pas seulement importantes dans le milieu de la traite des fourrures lié à Montréal par l'intermédiaire de la CNO et de l'AFC, mais aussi au sein de la société anichinabée et de son paysage familial ${ }^{82}$. En outre, il se peut bien que les rites religieux catholiques aient été utilisés pour renforcer les notions de parenté et de réciprocité aux fondements anichinabés ${ }^{83}$.

\section{DISCUSSION}

En 2012, Jacqueline Peterson, réputée ethnohistorienne de la traite des fourrures et des Métis, a passé en revue plus de trois décennies de recherche sur les communautés de traite des fourrures dans la région des Grands Lacs. À la suite de cet examen, elle a conclu que les Grands Lacs n'étaient pas le lieu de naissance des Métis de la rivière Rouge et qu'ils n'avaient pas non plus donné naissance à un groupe ethnique distinct,

81. Jennifer S. H. Brown, «Kinship Shock for Fur Traders and Missionaries: The Cross-Cousin Challenge", dans An Ethnohistorian in Rupert's Land: Unfinished Conversations (Edmonton, Athabasca University Press, 2017), p. 137-144; Laura Peers et Jennifer S. H. Brown, "There is no End to Relations among the Indians" : Ojibwa Families and Kinship in Historical Perspective», The History of the Family, 4 (2000), p. 529-555.

82. Le terme kinscape, que nous rendons par paysage familial, a d'abord été utilisé par Sami Lakomäki, spécialiste de l'histoire des Shawnees, pour décrire un espace social et géographique dans lequel des réseaux de parenté se chevauchant rayonnaient à partir de chaque communauté, reliant chacune à des dizaines d'autres, proches ou éloignées. Suivant cette vision du monde des Shawnees, la mobilité était considérée comme une composante nécessaire de leur monde et non comme une force perturbatrice. Sami Lakomäki, Gathering Together: The Shawnee People Through Diaspora and Nationhood, 1600-1870 (New Haven, Yale University Press, 2014), p. 229-230.

83. T. M. Schenck, All Our Relations..., p. 35-43. Pour une analyse étoffée du clan anichinabé et de sa structure sociopolitique, voir Theresa M. Schenck, "The Voice of the Crane Echoes Afar", The Sociopolitical Organization of the Lake Superior Ojibwa, 1640-1855 (New York et Londres, Garland Publishing Inc., 1997), p. 55-98. 
d'ascendance et de culture mixtes, comme les Métis de la rivière Rouge ${ }^{84}$. Elle s'opposait aux efforts actuels visant à faire l'amalgame entre deux histoires: celles de deux sociétés distinctes vivant de la traite des fourrures établies de part et d'autre de la ligne laurentienne de partage des eaux. Certes, les deux populations se connaissaient et partageaient des caractéristiques et des origines communes, mais leur développement respectif demeure largement distinct. Il s'agit de sociétés distinctes qui se livraient toutes deux à la traite des fourrures et qui ont eu, pendant un certain temps, un ancrage commun à Montréal et dans la vallée du Saint-Laurent en général. Mais si les communautés du bassin versant des Grands Lacs et du Saint-Laurent s'adonnant à la traite des fourrures n'étaient pas les mêmes que leurs homologues métisses des plaines de la baie d'Hudson, comment devrait-on comprendre cette société lacustre persistante établie plus à l'est? Que nous révèle l'analyse d'une collectivité particulière de la traite des fourrures dans les Grands Lacs - analyse effectuée «à partir du bas » dans le présent article - au sujet des personnes et des familles qui la composaient?

La première observation évidente, basée sur cette analyse ciblée des personnes participant aux rites sacramentels catholiques à La Pointe en 1835, est que les liens de parenté et les liens rituels décrits ici se sont répliqués d'un bout à l'autre des communautés franco-anichinabées du bassin hydrographique des Grands Lacs et du fleuve Saint-Laurent vivant de la traite des fourrures. Partout dans le pays du commerce des fourrures, à l'est de la ligne laurentienne de partage des eaux, on trouve parmi le personnel de la traite des fourrures des gens présentant des profils complexes, comme celui de Joseph Default - sa mère, sa belle-mère et la grand-mère de sa femme étaient Anichinabées; sa femme, son beau-père et lui étaient FrancoAnichinabés, tandis que son père, ses grands-parents paternels et le grandpère de sa femme étaient apparemment d'origine canadienne-française. Au début du XIX ${ }^{\mathrm{e}}$ siècle, les familles Dufault, Côté, Bélanger et surtout Cadotte avaient de multiples liens de parenté avec les populations anichinabées voisines et quantité de liens conjugaux et symboliques entre elles, tout en maintenant des liens familiaux, culturels et commerciaux avec Montréal et les vieilles paroisses canadiennes-françaises de la vallée du Saint-Laurent pourvoyeuses de voyageurs. Ces tendances continues en matière de parenté symbolique et réelle entre les populations canadienne-française, franco-

84. Jacqueline Peterson, «Red River Redux: Métis Ethnogenesis and the Great Lakes Region», dans Nicole St-Onge, Carolyn Podruchny et Brenda Macdougall, dir., Contours of a People: Métis Family, Mobility and History (Norman, OK, University of Oklahoma Press, 2012), p. 30. 
anichinabée et anichinabée contrastent fortement avec les tendances observées dans le cas des communautés de l'intérieur des Grandes Plaines se livrant à la chasse commerciale au bison ${ }^{85}$.

En nous basant sur ce travail initial concernant les familles de La Pointe, nous émettons l'hypothèse que la communauté de la traite des fourrures du bassin des Grands Lacs et de la vallée du Saint-Laurent n'était pas métisse de la même manière que la nation michief du bassin de la baie d'Hudson. Au début du XIX ${ }^{\mathrm{e}}$ siècle, ces peuples des Plaines s'étaient déclarés distincts de leur parenté amérindienne, canadienne-française ou écossaise, et ils fonctionnaient comme une entité distincte. En termes de parenté et de paysage familial, ces Métis des Plaines de l'Ouest étaient de plus en plus endogames dans leurs choix de conjoints; les Métis des Plaines chassant le bison avaient en effet tendance à épouser des Métis des Plaines chassant le bison de préférence aux Européens, aux Cris, aux Assiniboines ou autres tribus des Plaines $^{86}$. Comme l'a fait remarquer l'éminent marchand de fourrures de la CNO William McGillivray, dans une lettre écrite le 14 mars 1818 à la suite d'une enquête sur la bataille de la Grenouillère de 1816 (également connue sous le nom de massacre de Seven Oaks), «ils se considèrent tous comme membres d'une tribu indépendante d'Autochtones, ayant droit à la propriété du sol, à leur propre drapeau et à la protection du gouvernement britannique». McGillivray poursuit: "Les sang-mêlé sous les dénominations de Bois-Brûlés et de Métifs forment depuis longtemps une tribu d'Indiens séparée et distincte ${ }^{87}$.» Les Michiefs de l'Ouest occupaient un créneau socioéconomique distinct, à grande échelle, axé sur la chasse commerciale au bison, et celui-ci engendrait un mode de vie unique, des mœurs sociales et des principes de gouvernance distincts ainsi que des marqueurs culturels propres au groupe, comme un drapeau.

Inversement, la particularité de la population franco-anichinabée catholique qui vivait à l'est de la ligne laurentienne de partage des eaux et s'adonnait à la traite des fourrures ne tient pas à son caractère distinct de celui de ses voisins canadiens-français et anichinabés, mais plutôt à son intégration très réussie dans les deux populations. Au début du XIX siècle, les descen-

85. Voir par exemple les types de famille décrits dans Martha Harroun Foster, We Know Who We Are: Métis Identity in a Montana Community (Norman, OK, University of Oklahoma Press, 2016).

86. D. N. Sprague et R. P. Frye, The Genealogy of the First Métis Nation: The Development and Dispersal of the Red River Settlement, 1820-1900 (Winnipeg, Pemmican Publications, 1983).

87. Traduction d'un extrait du «Statement of William McGillivray to W. B. Coltman, March 14, 1818 ", cité par Gerhard J. Ens, "The Battle of Seven Oaks and the Articulation of a Métis National Tradition, 1811-1849", dans N. St-Onge, C. Podruchny et B. Macdougall, dir., Contours of a People..., p. 102-103. Voir aussi Adam Gaudry, «Métis», dans L'Encyclopédie canadienne, <https: / /www.thecanadianencyclopedia.ca/fr/article/metis $>$. 
dants des Bélanger, Cadotte, Côté et Dufault étaient tous membres d'un double réseau de parenté: un réseau centré sur les Anichinabés des Grands Lacs par l'entremise de leurs grands-mères, de leurs mères et parfois de leurs épouses; et un réseau franco-catholique issu des anciennes paroisses du Saint-Laurent pourvoyeuses de voyageurs qui ont produit des générations d'hommes franco-catholiques participant à une économie de la fourrure axée sur Montréal. Grâce à leur appartenance multigénérationnelle à deux sociétés bien établies, ces familles s'adonnant à la traite des fourrures ont pu participer pleinement à l'économie des fourrures des Grands Lacs et du Saint-Laurent en tant que marchands, voyageurs, employés de postes ou, pendant un certain temps, pêcheurs commerciaux.

Il s'agissait d'une adaptation efficace à une économie des pelleteries durable qui s'étendait sur de vastes régions géographiques et faisait appel à divers rôles professionnels essentiels. Ces personnes et leurs familles ont été les pivots très mobiles de cette entreprise complexe qui a connu une longue existence.

Les employés canadiens-français de la traite des fourrures et leurs épouses anichinabées venaient de sociétés patrilinéaires et virilocales où la parenté jouait un rôle essentiel. Les deux sociétés comprenaient clairement les obligations des liens de parenté et des liens de parenté symbolique. Ces réseaux de parents dans le contexte de la traite des fourrures se complétaient plutôt que de se contredire. Ces doubles réseaux ont permis aux familles de commerçants de fourrures de fonctionner facilement avec des parents résidant dans les centres de traite des fourrures de La Pointe, de Michilimackinac et de Sault-Sainte-Marie, tout en maintenant des relations continues avec les familles résidant le long de la vallée du Saint-Laurent et celles vivant dans les communautés anichinabées du lac Supérieur. Il s'agissait d'un monde liminal, soigneusement construit, axé sur les membres de la famille, reposant sur une économie de la traite des fourrures qui reliait Montréal et les paroisses environnantes à la population productrice de fourrures et de poisson des Pays d'en Haut. Il s'agissait également d'un monde fondé sur une compréhension mutuelle des obligations envers la famille nucléaire et la famille élargie et de leur importance capitale pour le bien-être continu de la collectivité dans son ensemble.

\section{OBSERVATIONS FINALES}

La présente analyse des activités religieuses et professionnelles de quatre familles donne également une idée des perceptions des Anichinabés à l'égard de ces Franco-Anichinabés. Ces derniers étaient distincts, mais 
tout de même parents, et ils étaient assez proches et assez appréciés pour que, dans les traités de 1826 et de 1837, les chefs négociateurs fassent valoir que des dispositions spéciales devaient être prises pour assurer leur bien-être continu dans un monde en évolution. Certains auteurs ont soutenu que ces membres «métis» ou "de sang mêlé » de la tribu avaient escroqué les Indiens pendant les négociations du traité, mais cette affirmation fait entorse à la vérité ${ }^{88}$. Au moment des traités américains des années 1820 et 1830, les Anichinabés des Grands Lacs avaient depuis plusieurs générations des contacts suivis avec la population franco-catholique de la vallée du Saint-Laurent. Il restait peu d'illusions de part et d'autre de cette équation particulière de la traite des fourrures.

L'inquiétude pour le bien-être de cette population découlait peut-être d'une façon de percevoir ces Anichinabés franco-catholiques qui correspondait à une vision du monde anichinabée traditionnelle. Selon celle-ci, les familles Cadotte, Bélanger, Côté et Dufault étaient issues de clans ou dodems anichinabés, c'est-à-dire de familles patrilinéaires apparentées qui se considéraient comme les descendants d'un ancêtre commun, avec des caractéristiques distinctives et des obligations socio-économiques particulières ${ }^{89}$. Les quatre familles étudiées ici n'étaient pas liées à des ancêtres communs revêtant une figure animale, mais elles avaient effectivement des origines communes: la vallée du Saint-Laurent, la religion catholique et la langue française. Il s'agissait peut-être là d'un nombre suffisant de points communs pour que leurs beaux-parents anichinabés les considèrent collectivement, eux et leurs familles, comme une série de dodems émergents.

Dans la société anichinabée, les règles relatives à la parenté et aux réseaux de parenté régissent tous les aspects de la vie d'un individu, comme l'historienne Heidi Bohaker l'a largement démontré ${ }^{0}$. Ils n'étaient pas si différents des autres clans anichinabés qui s'unissaient eux aussi aux $\mathrm{XVIII}^{\mathrm{e}}$ et XIX ${ }^{\mathrm{e}}$ siècles, à mesure que cette population s'étendait depuis la région de Sault-Sainte-Marie vers la partie sud du lac Supérieur. Plus pré-

88. On trouvera un exemple de ce point de vue dans Wub-E-Ke-Niew, We Have the Right to Exist: A Translation of Aboriginal Indigenous Thought: The First Book Ever Published from an Ahnishinahbcétjibway Perspective (New York, Black Thistle Press, 1995).

89. T. M. Schenck, "The Voice of the Crane Echoes Afar", p. 29-35; Charles E. Cleland, Rites of Conquest: The History and Culture of Michigan's Native American (Ann Arbor, University of Michigan Press, 1992), p. 41-54.

90. Heidi Bohaker, "Nindoodemag": The Significance of Algonquin Kinship Networks in the Eastern Great Lakes Region, 1600-1701 ", The William and Mary Quarterly, 63, 1 (janvier 2006), p. 23-52. Heidi Bohaker, «Anishinaabe Toodaims: Contexts for Politics, Kinship, and Identity in the Eastern Great Lakes ", dans Carolyn Podruchny et Laura Peers, dir., Aboriginal and Fur Trade Histories: Gathering Places (Vancouver, University of British Columbia Press, 2010), p. 93-118. 
cisément, les familles Cadotte et Dufault étaient liées aux familles de chefs du lac Supérieur, en particulier à l'éminent clan anichinabé de la Grue. Il n'est donc pas impossible qu'elles aient été considérées comme des sousclans associés à la Grue. Cette filiation pourrait expliquer pourquoi certains membres de ces familles, même après avoir accepté un certificat de concession de terres destinées aux sang-mêlé en 1839, sont restés avec les tribus et sont finalement devenus des membres inscrits ou réinscrits de la tribu. Ils constituaient des membres respectés et appréciés d'un monde autochtone des Grands Lacs. Cette tentative de normalisation et d'intégration de ces familles franco-anichinabées présentes dans l'ensemble des Grands Lacs aux XVIII et ${ }^{e}$ XIX $^{e}$ siècles aurait eu un sens. Il existe dans les archives historiques des indices probants selon lesquels une stratégie similaire a été utilisée dans le pays de l'Illinois pour intégrer des familles commerçantes apparentées en formant, à leur intention, une sixième division au sein de la confédération shawnee ${ }^{91}$.

D'autres membres des familles examinées ont choisi de rester ou de retourner dans la vallée du Saint-Laurent au crépuscule de la période de la traite des fourrures, soit au moment de l'accélération de l'ère des réserves aux États-Unis ${ }^{92}$. Mais, au milieu du XIX ${ }^{\mathrm{e}}$ siècle, une partie de la population de la vallée du Saint-Laurent était sans terre et de plus en plus appauvrie. Le commerce du bois était apparemment insuffisant pour compenser la disparition d'une économie basée sur la traite des fourrures centrée sur Montréal, les sombres perspectives agricoles et les conditions de vie inimaginables de la classe ouvrière en pleine expansion à Montréal ${ }^{93}$. Rester au sud des Grands Lacs paraissait peut-être plus sage en ces années incertaines, avec des parents tout autour, dans une géographie familière où il y avait moyen de trouver du travail lié à la pêche commerciale et aux débuts du commerce du bois.

Mais il est bien possible que la vérité absolue soit plus profonde que cela. Dans les années 1840, les descendants des quatre couples fondateurs étudiés dans cet article semblent avoir été pleinement acceptés en tant

91. Robert Englebert, "Colonial Encounters and the Changing Contours of Ethnicity : Pierre-Louis de Lorimier and Métissage at the Edges of Empire», Ohio Valley History, 18, 1 (printemps 2018), p. 57.

92. Par exemple, les descendants des familles Dufault, Cadotte et Lacombe sont revenus ou sont restés dans la vallée du Saint-Laurent, où ils ont élevé leur propre famille. Ce geste marque la fin d'une économie basée sur la traite des fourrures reliant le Saint-Laurent au sud des Grands Lacs. Il a arrêté la circulation pelletière et rompu les liens familiaux qui existaient depuis des générations dans le bassin des Grands Lacs et du fleuve Saint-Laurent.

93. Du milieu à la fin du XIX ${ }^{\mathrm{e}}$ siècle, Montréal enregistra des taux de mortalité supérieurs à ceux de n’importe quelle autre ville de l'Amérique du Nord britannique. Voir Bettina Bradbury, Familles ouvrières à Montréal: âge, genre et survie quotidienne pendant la phase d'industrialisation (Montréal, Boréal, 1995 [1993]). 
qu'Anichinabés, du moins par la tribu, sinon par l'État américain. Leurs quatre noms de famille se retrouvent dans les nombreux clans et sousclans qui ont inspiré la cosmologie et le paysage familial des Anichinabés au XIX ${ }^{\mathrm{e}}$ siècle. Une analyse plus poussée des registres de catholicité de la mission de La Pointe et des missions catholiques avoisinantes révélerait si les pratiques traditionnelles des Anichinabés, comme les mariages entre cousins croisés, se sont poursuivies entre les familles élargies franco-anichinabées et les membres des autres clans ${ }^{94}$. Des pratiques de ce genre indiqueraient qu'au moins quelques segments des anciennes collectivités vivant de la traite des fourrures autour du lac Supérieur s'intégraient continuellement dans le monde tribal après l'établissement des réserves. Les patronymes de toutes les familles dont il a été question dans cet article figurent assurément sur les listes de recensement des Indiens des ÉtatsUnis pour les divisions administratives du Wisconsin, du Minnesota et du Dakota du Nord pendant une bonne partie du $\mathrm{XX}^{\mathrm{e}}$ siècle.

(Traduction: André LaRose) 